\title{
WADING THROUGH THE QUAGMIRE OF SCHMIDT-PLATE COORDINATE SYSTEMATICS
}

\author{
C.-L. LU ${ }^{1}$, I. PLATAIS ${ }^{1}$, T.M. GIRARD ${ }^{1}$, \\ V. KOZHURINA-PLATAIS ${ }^{1}$, W.F. VAN ALTENA ${ }^{1}$, \\ C.E. LÓPEZ ${ }^{2}$ AND D.G. MONET ${ }^{3}$ \\ ${ }^{1}$ Yale University Observatory, New Haven, CT 06511, USA \\ ${ }^{2}$ Félix Aguilar Observatory, San Juan, Argentina \\ ${ }^{3}$ US Naval Observatory, Flagstaff, AZ 86002, USA
}

\section{Magnitude-dependent Errors}

We attempted to quantify the magnitude-dependent systematics in a sample of Schmidt plates by comparison to positions from the Yale/San Juan Southern Proper Motion program which offers star positions and absolute proper motions down to $B=18$ with a mean density of about 50 stars per square degree and a positional accuracy of $0.1^{\prime \prime}$ (Platais et al. 1995).

The Schmidt-plate coordinates used in this study are those from the USNO_A0.9 all-sky catalog (Monet 1996). In total for 53 SPM fields we extracted 46 corresponding Schmidt survey fields (30 UKST and 16 Palomar) with more than 1000 stars in common. First, the variations in differences "SPM-USNO_A0.9" with position were removed using the infinite overlapping circle method (Taff et al. 1990). These variations undoubtedly were inherited from the Guide Star Catalog which served as the reference catalog. Second, the post-geometric-correction residuals plotted as a function of magnitude clearly indicate a trend with magnitude. Morrison et al. (1996) reported the existence of fixed-pattern, magnitude-dependent systematics in the Guide Star Catalog positions. In addition to that, our comparisons unambiguously show a plate-to-plate variable magnitude equation.

\section{Conclusions}

Magnitude-dependent systematics, which vary from plate to plate, are present in Schmidt-plate based coodinates at a level indicated in Figure 

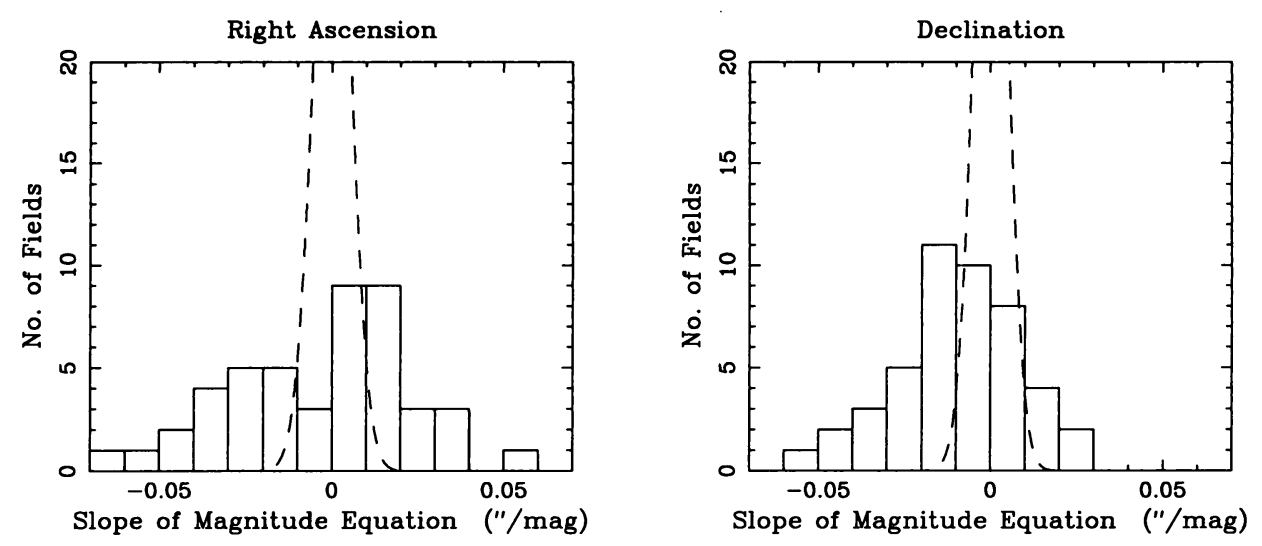

Figure 1. The distribution of fitted magnitude-equation slopes for all 46 Schmidt fields. The dashed curve shows the expected distribution in the case of no magnitude equation.

1. Assuming the geometrical distortions are corrected using a reference catalog at $B \approx 11$, the magnitude-dependent offsets will be largest at the faint end. Similarly, the absolute proper motions are expected to suffer from magnitude equation, although this time for the bright stars. If ignored, the magnitude equation has obvious implications for faint positional reference catalogs and for proposed galactic structure studies.

\section{References}

Monet, D. G. 1996. DDA meeting (April 15-17) held in Washington, DC, abstract

Morrison, J. E., Röser, S., Lasker, B. M., Smart, R. L. and Taff, L. G., 1996. Astron. J., $111,1405$.

Platais, I., Girard, T. M., van Altena, W. F., Ma, W.-Z., Lindegren, L., Crifo, F. and Jahreiß, H., 1995. Astron. Astrophys., 304, 141.

Taff, L. G., Bucciarelli, B. and Lattanzi, M. G., 1990. Astrophys. J., 361, 667. 